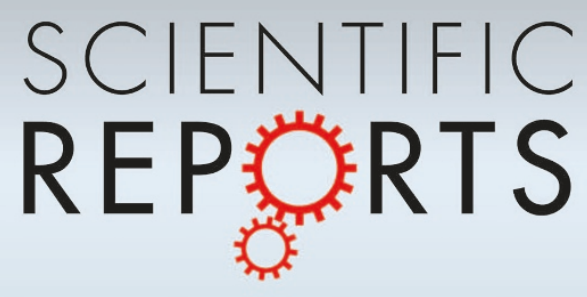

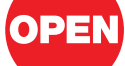

SUBJECT AREAS: ANTIBODY GENERATION IMMUNOLOGICAL TECHNIQUES MOLECULAR ENGINEERING BIOLOGICAL TECHNIQUES

Received

20 November 2012

Accepted

19 December 2012

Published

23 January 2013

Correspondence and requests for materials should be addressed to J.T.A. (j.t.andersen@ imbv.uio.no)

* Current address: arGEN-X bvba,

Technologiepark 30, 9052 Ghent, Belgium.

† Current address: NSpired BVBA, Belgium.

\section{Selection of Nanobodies that Target Human Neonatal Fc Receptor}

\author{
Jan Terje Andersen ${ }^{1,2}$, Maria Gonzalez-Pajuelo ${ }^{3}$, Stian Foss ${ }^{1,2}$, Ole J. B. Landsverk', Débora Pinto ${ }^{3}$, \\ Alexander Szyroki ${ }^{3}$, Hans J. de Haard ${ }^{3 *}$, Michael Saunders ${ }^{3 *}$, Peter Vanlandschoot ${ }^{3+}$ \& Inger Sandlie ${ }^{1,2}$
}

${ }^{1}$ Centre for Immune Regulation (CIR) and Department of Molecular Biosciences, University of Oslo, P.O. box 104, 1N-0371 Oslo, Norway, ${ }^{2} \mathrm{CIR}$ and Department of Immunology, Oslo University Hospital, University of Oslo, P.O. Box 4956 Nydalen, Postbox N0424, Oslo, Norway, ${ }^{3}$ Ablynx NV, Technologiepark 21, 9052 Ghent Zwijnaarde, Belgium.

FcRn is a key player in several immunological and non-immunological processes, as it mediates maternal-fetal transfer of IgG, regulates the serum persistence of IgG and albumin, and transports both ligands between different cellular compartments. In addition, FcRn enhances antigen presentation. Thus, there is an intense interest in studies of how FcRn binds and transports its cargo within and across several types of cells, and FcRn detection reagents are in high demand. Here we report on phage display-selected Nanobodies that target human FcRn. The Nanobodies were obtained from a variable-domain repertoire library isolated from a llama immunized with recombinant human FcRn. One candidate, Nb218-H4, was shown to bind FcRn with high affinity at both acidic and neutral $\mathrm{pH}$, without competing ligand binding and interfering with FcRn functions, such as transcytosis of IgG. Thus, Nb218-H4 can be used as a detection probe and as a tracker for visualization of FcRn-mediated cellular transport.

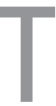
he neonatal $\mathrm{Fc}$ receptor $(\mathrm{FcRn})$ was originally isolated from the intestinal epithelium of neonatal rats and shown to mediate uptake of IgG derived from the mother's milk ${ }^{1,2}$, thereby its name. However, a large body of evidence has shown that FcRn is not restricted to neonatal life but is expressed in a variety of tissues and cell types at all stages of life, as reviewed elsewhere ${ }^{3,4}$. A diverse set of FcRn functions at different body sites have just begun to emerge. The most studied function is its role as a serum half-life regulator, as FcRn extends the serum persistence of IgG subclass antibodies and albumin to three weeks in humans ${ }^{5-9}$. Responsible is a cellular recycling mechanism that takes place in hematopoietic cells as well as endothelial cells lining blood vessels ${ }^{10-12}$. Within these cells, FcRn resides predominantly in acidified intracellular compartments where the low $\mathrm{pH}$ triggers binding of IgG and albumin to FcRn. This results in recycling of the complex back to the cell surface where exposure to the near neutral $\mathrm{pH}$ of the blood triggers release of the ligands from the receptor. The strict $\mathrm{pH}$ dependence of both interactions is fundamental for efficient recycling and thereby rescue from intracellular degradation.

In addition, FcRn transports IgG across different cellular barriers such the mucosal epithelium lining the intestine and the alveolar surfaces ${ }^{13,14}$, and delivers IgG from the mother to the fetus via the placenta ${ }^{15,16}$. The mechanism secures passive immunity of the newborn in a critical phase of early life. Furthermore, FcRn has been found to collaborate with the classical Fc $\gamma$ receptors expressed on immune cells in orchestration of uptake and processing of IgG-immune complexes, leading to antigen presentation and induction of T cell immunity ${ }^{17,18}$.

FcRn is a major histocompatibility complex (MHC) class I-related transmembrane trafficking receptor. It is a heterodimer with an evolutionally distinct heavy chain (HC) non-covalently associated with the $\beta 2$-microglobulin light chain, which is common to most MHC class I family molecules ${ }^{19,20}$. For all functions, $\mathrm{pH}$-dependent binding to both ligands is fundamental, binding at acidic $\mathrm{pH}(6-6.5)$, and releases or no binding at neutral $\mathrm{pH}$ $(7.4)^{6,19,21}$. IgG and albumin bind to separate binding sites on the FcRn HC in a non-cooperative fashion ${ }^{21-24}$. X-ray crystallography and site-directed mutagenesis studies have revealed that histidine residues located at the $\mathrm{C}_{\mathrm{H}} 2-$ $\mathrm{C}_{\mathrm{H}} 3$ elbow region of the IgG Fc (H310 and H435) are central for IgG binding, ${ }^{6,20}$. At acidic $\mathrm{pH}$ their imidazole groups are positively charged and facilitate interaction with negatively charged residues located within the $\alpha_{2-}$ domain of the receptor (E115 and E116 in humans, and E117 and E118 in rats), while at pH 7.4, the imidazole rings are neutral and thereby do not interact with FcRn. Similarly, three histidines within the C-terminal domain III of albumin (H464, H510 and H535) are engaged in binding to FcRn at a site opposite the IgG binding site ${ }^{21}$. In addition, H166 of the $\alpha_{2}$-domain of FCRn stabilizes a flexible surface-exposed loop within the $\alpha 1$-domain (residue 51-60) of hFcRn in a pH-dependent way ${ }^{21,22}$. Thus, histidines are crucial for $\mathrm{pH}$-dependent binding of FcRn to both ligands. 
A

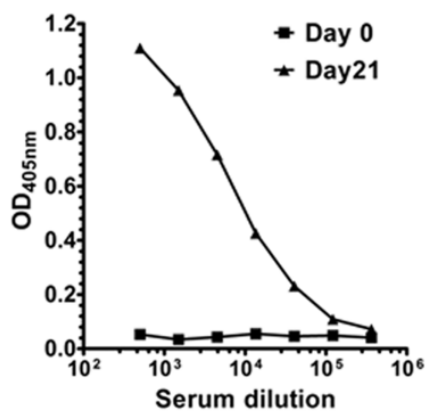

B

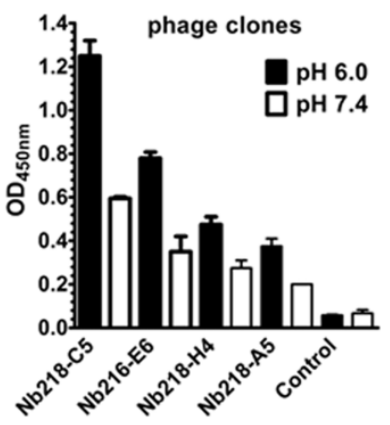

Figure $1 \mid$ Immunization of a llama and selection of anti-hFcRn phage particles. (A) Serum samples collected before and after immunization of a llama with recombinant hFcRn. Serial dilutions of sera were incubated in $\mathrm{hFcRn}$ coated ELISA wells and bound antibodies were detected using a anti-llama IgG from mouse followed by an anti-mouse IgG secondary antibody from rabbit. (B) Representative ELISA results showing binding of selected phage clones to $\mathrm{hFcRn}$ at $\mathrm{pH} 6.0$ and $\mathrm{pH}$ 7.4. The numbers given represent the mean of duplicates.

FcRn function is critical for controlling and optimizing pharmacokinetics and bioavailability of monoclonal IgG and IgG Fc-fused therapeutics as well as albumin-fused or conjugated therapeutics ${ }^{25,26}$. Still, major questions regarding the cellular distribution of FcRn and how it transports both its ligands across or within different cell types remain to be addressed at a molecular and cellular level. To study the biology of FcRn in health and disease, methods to detect its presence and transport are needed, and in particular, detection reagents other than anti-FcRn IgG antibodies, which may interfere with FcRn function, are preferred.

This report describes the generation of FcRn probes from a combinatorial library of light chain devoided IgG variable regions obtained from a llama immunized with recombinant soluble $\mathrm{hFcRn}$ using phage display. Such variable $\mathrm{HC}$ only (VHH) antibodies are naturally occurring in Camelidae and their entire binding unit is termed Nanobodies ${ }^{\circledR}(\mathrm{Nb})^{27}$. We identified Nbs with specificity for folded hFcRn, which do not interfere with $\mathrm{pH}$-dependent binding to IgG or albumin. Importantly, $\mathrm{Nb}$ binding to hFcRn was not affected by $\mathrm{pH}$ and did not affect FcRn-mediated transcellular transport of IgG. The Nbs could also be directly conjugated to an enzyme or fluorochromes without losing FcRn binding activity. Thus, the Nbs selected are attractive tools for studying FcRn biology.
A

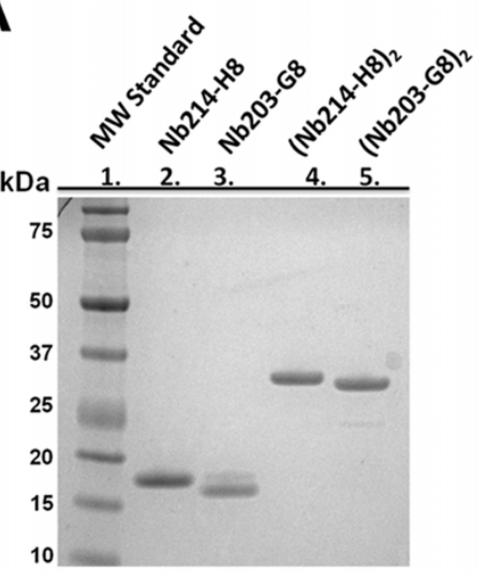

B
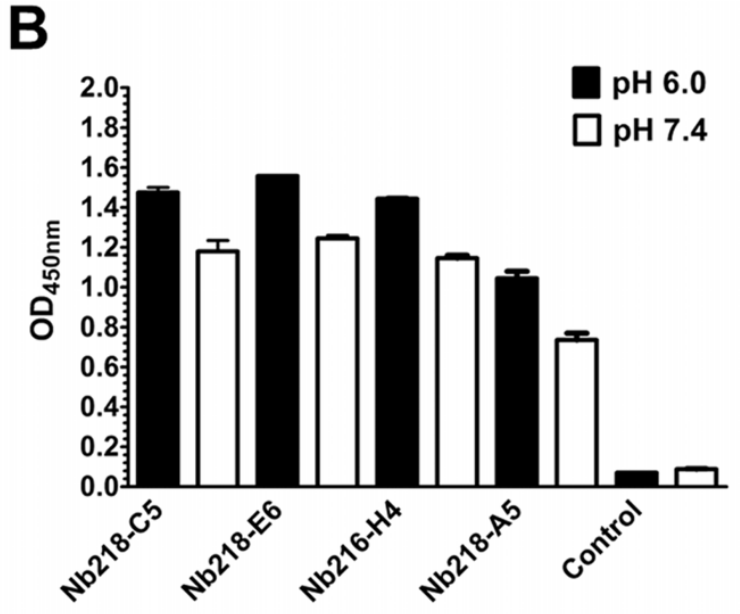

$E$

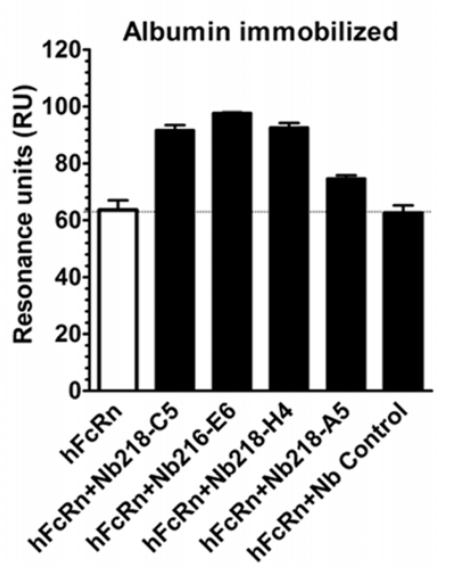

Figure 2 Bacterial production of Nbs and binding to hFcRn. (A) Nb candidates were produced in E. coli and subsequently purified and analyzed by $12 \%$ SDS-PAGE. Lane 1 shows a molecular weight (MW) standard, lane 2 shows Nb218-H4, lane 3 shows Nb203-G8, lane 4 shows (Nb218-H4) 2 , and lane 5 $(\mathrm{Nb} 203-\mathrm{G} 8)_{2}$. (B) Binding of GST-tagged hFcRn to the Nb variants captured on an anti-c-Myc antibody at pH 6.0 and pH 7.4. Bound receptor was visualized using an HRP-conjugated anti-GST antibody. (C) Binding of the Nb variants to human $\beta 2$-microglobulin coated in wells and detected with an anti-c-Myc antibody. An HRP-conjugated anti-human $\beta 2$-microglobulin antibody was included as a positive control. The numbers given represent the mean of triplicates. (D-E) Anti-hFcRn Nbs bind hFcRn independently of the presence of the ligands. Representative SPR responses showing binding of hFcRn alone or in complex with anti-hFcRn Nbs or a control $\mathrm{Nb}$ to (D) human IgG and (E) human albumin immobilized on a CM5 sensor chip. Injections were done at $\mathrm{pH} 6.0$ at $25^{\circ} \mathrm{C}$ with a flow rate of $50 \mu \mathrm{l} / \mathrm{min} . n=3-4$. All data are presented as mean \pm s.d. 

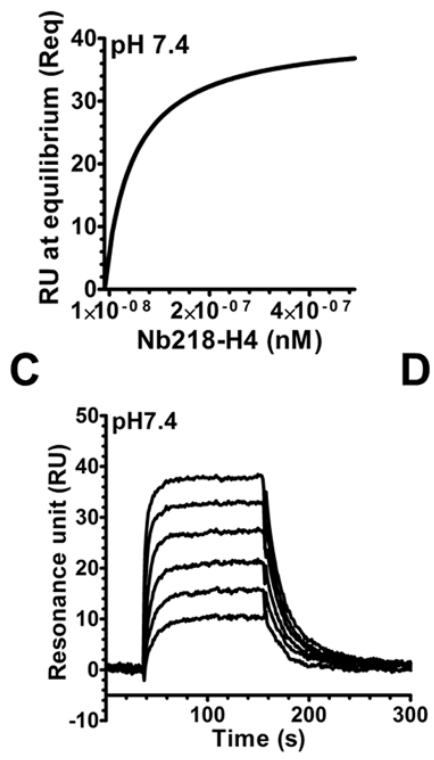

D
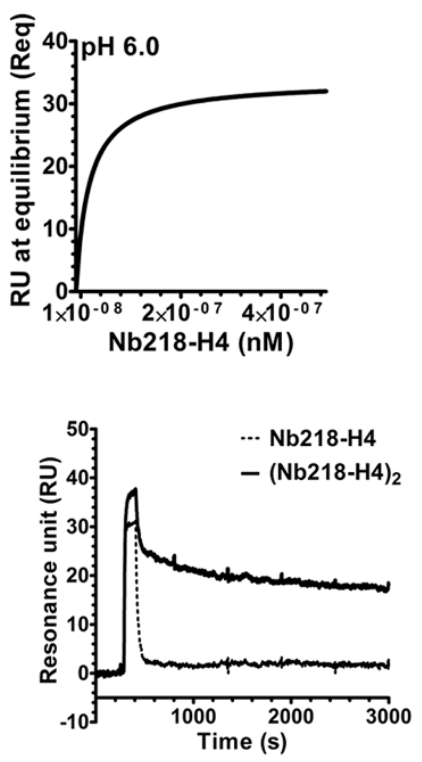

Figure $3 \mid$ Binding properties of monomeric and dimeric forms of antihFcRn Nb218-H4. Serial dilutions of Nb218-H4 injected over immobilized $\mathrm{hFcRn}$ at either $\mathrm{pH} 7.4$ or $\mathrm{pH}$ 6.0. The equilibrium binding responses obtained at (A) $\mathrm{pH} 7.4$ and (B) $\mathrm{pH} 6.0$ were fitted to a steady state affinity binding model supplied with the BIAevaluation software. (C) A representative sensorgram showing the overlays of serial dilutions of $\mathrm{Nb} 218-\mathrm{H} 4$ injected over immobilized hFcRn at $\mathrm{pH}$ 7.4. The derived binding kinetic values are presented in Table 1. (D) A representative sensorgram showing binding of equal amounts of monomeric $\mathrm{Nb} 218-\mathrm{H} 4$ and bivalent $(\mathrm{Nb} 218-\mathrm{H} 4)_{2}$ to immobilized $\mathrm{hFCRn}$ at $\mathrm{pH}$ 6.0. Injections were performed at $25^{\circ} \mathrm{C}$ with a flow rate of $50 \mu \mathrm{l} / \mathrm{min}$. $n=3-4$. All data are presented as mean \pm s.d.

\section{Results}

Immunization of llamas. One llama (L.glama) was immunized with purified soluble recombinant hFcRn over a period of 6 weeks. The induction of llama IgG antibodies directed towards $\mathrm{hFcRn}$ was demonstrated by ELISA on sera collected before and 21 days after immunization, (Figure 1A). Subsequently, a cDNA library encoding the in vivo matured variable domain repertoire of $\mathrm{HC}$ only antibodies (VHH) were prepared from isolated PBLs and draining lymph nodes biopsies, followed by cloning of the PCR-amplified fragments into a phage display library. The resulting library contained approximately $7-8 \times 10^{8}$ independent clones.

Phage display selection. To select for hFcRn-specific phages, the phage display library was panned on recombinant hFcRn coated by ELISA wells at $\mathrm{pH} 5.1$ followed by trypsin elution. After two rounds of selection, individual phages were isolated and screened for binding to hFcRn at $\mathrm{pH} 6.0$ and $\mathrm{pH} 7.1$ in ELISA. Phage particles were identified that bound hFcRn at both $\mathrm{pH}$ conditions. Binding of four of the selected phage clones is shown in Figure 1B, where the strongest binding response was seen for a phage clone $\mathrm{Nb} 218-\mathrm{C} 5$ followed by Nb216-E6, Nb218-H4 and Nb218-A5. All the clones showed a slightly better response at acidic than at neutral $\mathrm{pH}$.

Binding properties of selected anti-hFcRn Nanobodies. The variable domains from four isolated phage clones were sub-cloned into an expression vector designed for production of recombinant $\mathrm{Nbs}$ in E. coli. The cDNAs were cloned upstream of a C-terminal cMyc tag followed by a His ${ }_{6}$ tag. The purified Nbs was analyzed on SDS-PAGE gels and seen as distinct bands of approximately 15$17 \mathrm{kDa}$, corresponding to their expected molecular weights. A representative SDS-PAGE gel of the purified $\mathrm{Nb} 218-\mathrm{H} 4$ and a control variant with irrelevant specificity (Nb203-G8) is shown in Figure 2A.

To verify that the produced monomeric Nbs bound hFcRn, ELISA was performed with equal amounts of the Nbs captured on an anti cMyc mouse IgG1 antibody coated in wells (mIgG1 does not bind hFcRn). Receptor binding was confirmed by adding GST-tagged hFcRn followed by visualization using an HRP-conjugated antiGST antibody. All four Nbs were shown to bind the receptor at both $\mathrm{pH}$ conditions with a slightly better binding at $\mathrm{pH} 6.0$ (Figure 2B). $\mathrm{Nb} 218-\mathrm{C} 5, \mathrm{Nb} 216-\mathrm{E} 6$ and $\mathrm{Nb} 218-\mathrm{H} 4$ bound equally well, followed by $\mathrm{Nb} 218-\mathrm{A} 5$, while the negative $\mathrm{Nb}$ control did not bind.

$\mathrm{FcRn}$ is a heterodimer consisting of a $\mathrm{HC}$ that is folded up on $\beta 2$ microglobulin, which is shared between all MHC class I and related molecules. Thus, to be used as cellular detection probe for hFcRn, the selected Nbs must bind specifically to the HC only. To avoid selection of anti- $\beta 2$-microglobulin clones, selection steps were performed in the presence of human $\beta 2$-microglobulin in solution. To confirm that the generated $\mathrm{Nbs}$ bound specifically to the $\mathrm{HC}$, we also screened for binding towards human $\beta 2$-microglobulin by ELISA, and conclusively demonstrated that all four Nbs bound specifically to the hFcRn HC (Figure 2C). Furthermore, we confirmed by SPR that the anti-FcRn Nbs bound hFcRn at both $\mathrm{pH}$ conditions (Supplemental Fig. S1).

Selected Nbs bind hFcRn independently of the ligands. To investigate whether or not IgG and albumin bound to hFcRn affected binding of the $\mathrm{Nbs}$ at acidic $\mathrm{pH}$, we used SPR with immobilized human IgG1 and albumin on CM5 sensor chips and injected recombinant hFcRn alone or in complex with anti-FcRn Nbs at $\mathrm{pH}$ 6.0. None of the Nbs interfered with binding of the ligands to hFcRn, as additive binding to immobilized ligands was observed for all four $\mathrm{Nbs}$, while binding in the presence of $\mathrm{Nb}$ control was unaffected (Figure 2D-E).

Monomeric and bivalent Nb218-H4. The binding affinity of monomeric Nb218-H4 was determined by SPR by injection of serial dilutions over immobilized hFcRn at $\mathrm{pH} 6.0$ and $\mathrm{pH}$ 7.4. The resulting binding curves were either fitted to a steady state affinity binding model (Figure 3A-B) or a simple 1:1 Langmuir binding model (Figure 3C). Both kinetic binding models gave rise to similar estimated $\mathrm{KD}$ values of roughly $20 \times 10^{-9} \mathrm{M}$ at $\mathrm{pH} 6.0$, and a 2 -fold weaker affinity at $\mathrm{pH} 7.4$ (Table 1).

To improve apparent affinity through avid binding, we constructed a dimeric form of $\mathrm{Nb} 218-\mathrm{H} 4$. Two units were genetically fused via a glycine-serine linker. The purified $(\mathrm{Nb} 218-\mathrm{H} 4)_{2}$ variant as well as a dimeric negative control $\mathrm{Nb}$ were shown to migrate with the expected molecular sizes on a SDS-PAGE gel (Figure 2A). Then,

Table 1 | Binding kinetics of $\mathrm{Nb} 218-\mathrm{H} 4$

$\mathrm{Nb}$

$1: 1$ Langmuir model

Steady state model

\begin{tabular}{|c|c|c|c|c|c|c|c|}
\hline $\begin{array}{l}\mathrm{Nb} 218-\mathrm{H} 4 \\
\mathrm{Nb} 218-\mathrm{H} 4\end{array}$ & $\begin{array}{l}\mathrm{pH} \\
7.4 \\
6.0\end{array}$ & $\begin{array}{c}\mathrm{ka} \\
7.8 \mathrm{e}^{5} \pm 0.1 \\
12.4 \mathrm{e}^{5} \pm 0.2\end{array}$ & $\begin{array}{c}k d \\
4.1 \mathrm{e}^{-2} \pm 0.1 \\
2.6 \mathrm{e}^{-2} \pm 0.1\end{array}$ & $\begin{array}{l}\mathrm{KA} \\
1.9 \mathrm{e}^{7} \\
4.7 \mathrm{e}^{7}\end{array}$ & $\begin{array}{c}\mathrm{KD} \\
52.5 \mathrm{e}^{-9} \\
21.2 \mathrm{e}^{-9}\end{array}$ & $\begin{array}{c}\mathrm{KA} \\
1.8 \mathrm{e}^{7} \\
4.6 \mathrm{e}^{7}\end{array}$ & $\begin{array}{c}K D \\
55.6 \mathrm{e}^{-9} \\
22.0 \mathrm{e}^{-9}\end{array}$ \\
\hline
\end{tabular}


A
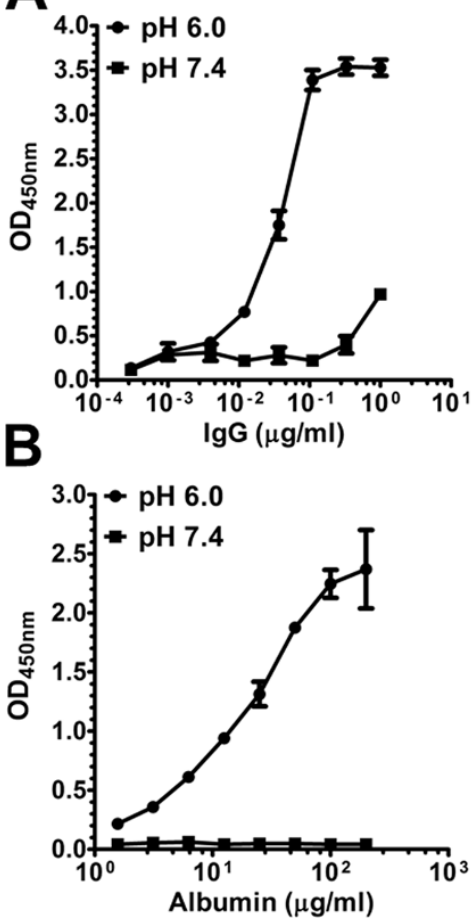
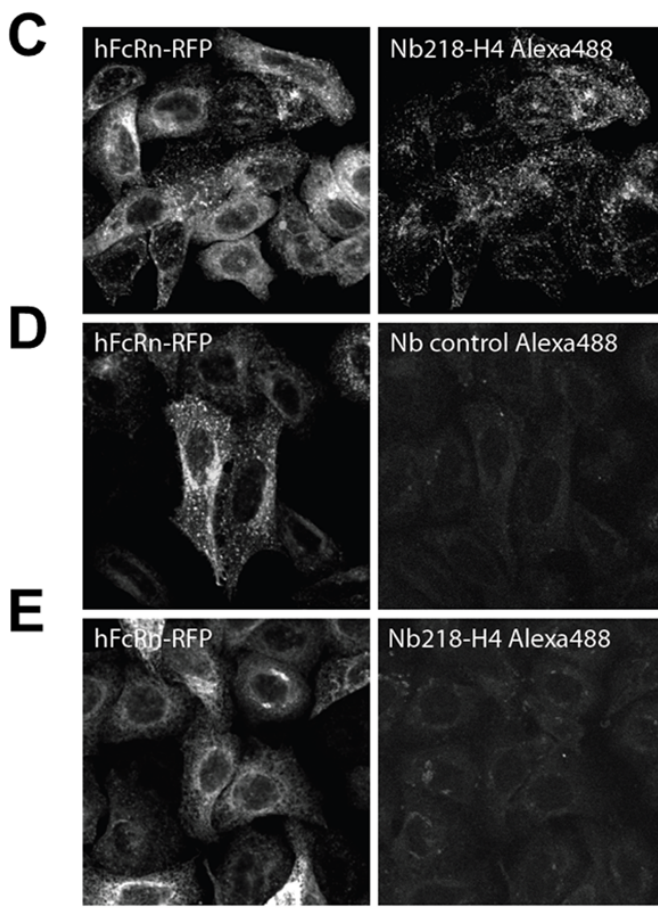

$\mathrm{Nb}$ control Alexa488
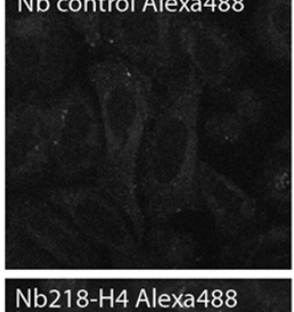

Nb218-H4 Alexa488

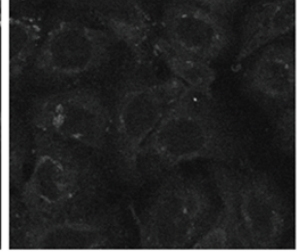

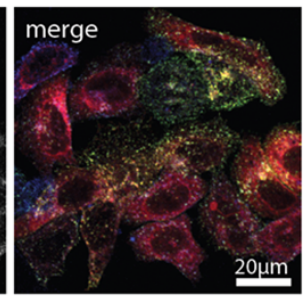

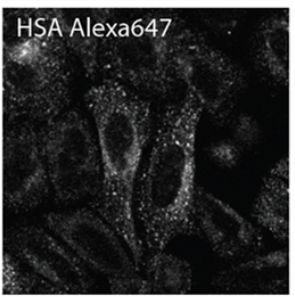

merge
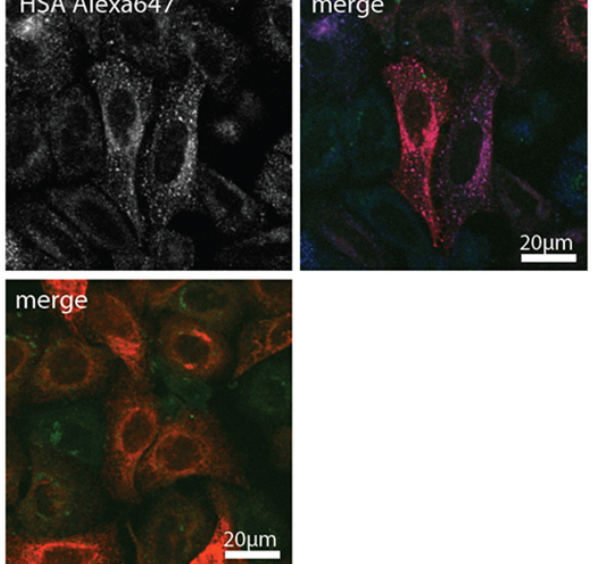

Figure $4 \mid$ Conjugation to Nb218-H4 and detection of hFcRn. Binding of hFcRn to titrated amounts of (A) human IgG1 and (B) albumin coated in wells. Binding of hFcRn to the ligands at $\mathrm{pH} 7.4$ and $\mathrm{pH} 6.0$ was visualized using HRP-conjugated $\mathrm{Nb} 218-\mathrm{H} 4$. The numbers given represent the mean of triplicates. HeLa cells transfected to over-express hFcRn (hFcRn-RFP and human $\beta 2$-microclobulin) were stained with (C) Alexa-conjugated (Nb218$\mathrm{H} 4)_{2}$ or (D) a control bivalent Nb. (C-D) Cells were also allowed to take up human albumin conjugated to Alexa647. (E) HeLa cells transfected with a plasmid encoding only the RFP-fused hFcRn HC and then incubated with Alexa-conjugated $(\mathrm{Nb} 218-\mathrm{H} 4)_{2}$ or a control $\mathrm{Nb}$. All cells were inspected by confocal microscopy as described in Material and Methods. $n=3$. All data are presented as mean \pm s.d.

equimolar amounts of monomeric and dimeric $\mathrm{Nb} 218-\mathrm{H} 4$ were injected over immobilized $\mathrm{hFcRn}$, which resulted in a considerably increased avidity of the dimer as demonstrated by a very slow dissociation phase compared with the monomer (Figure 3D).

Conjugation and visualization of hFcRn. To validate the utility of these $\mathrm{Nb}$ as detection probes, we then tested FcRn binding of Nb218$\mathrm{H} 4$ directly conjugated to an enzyme or fluorochrome. First, HRPconjugation to $\mathrm{Nb} 218-\mathrm{H} 4$ was shown not to disrupt binding to hFcRn, as the conjugate could be used as a sensitive detection probe in ELISA assays for $\mathrm{pH}$-dependent binding of FcRn to IgG and albumin (Figure 4A-B).

Next, we investigated whether Alexa488-conjugated (Nb218-H4) could be used for visualization of cellularly expressed hFcRn. HeLa cells were transfected with plasmids encoding RFP-fused hFcRn and human $\beta 2$-microglobulin, and the next day, cells were chased with Alexa488-conjugated $(\mathrm{Nb} 218-\mathrm{H} 4)_{2}$ or a bivalent $\mathrm{Nb}$ control. Only Alexa488-(Nb218-H4) 2 was shown to co-localize with hFcRn-RFP in intracellular compartments (Figure 4C). When Alexa647-conjugated human albumin was added in the presence of Alexa488-(Nb218$\mathrm{H} 4)_{2}$, RFP-hFcRn was shown to localize with both $(\mathrm{Nb} 218-\mathrm{H} 4)_{2}$ and albumin (Figure 4D).

Furthermore, HeLa cells transfected with a plasmid encoding only the RFP-fused hFcRn HC showed retention of the HC in the endoplasmatic reticulum, which is in agreement with previous findings demonstrating that proper folding of the FcRn HC is completely dependent on co-expression of $\beta 2$-microglobulin ${ }^{28}$. Importantly, $(\mathrm{Nb} 218-\mathrm{H} 4)_{2}$ did not co-stain with RFP-hFcRn in cells transfected with the HC only (Figure $4 \mathrm{E})$. Thus, anti-FcRn $(\mathrm{Nb} 218-\mathrm{H} 4)_{2}$ binds only to folded heterodimeric $\mathrm{hFcRn}$. In addition, we confirmed that Alexa488-conjugated (Nb218-H4) ${ }_{2}$ could be used to stain FcRn positive cells by flow cytometry (Supplemental Fig. S2).
FcRn-mediated transcytosis of IgG. Finally, we addressed whether the presence of $\mathrm{Nb} 218-\mathrm{H} 4$ would affect $\mathrm{FcRn}$-mediated transcytosis of human IgG1 across a monolayer of T84, a polarized human epithelial cell line known to express endogenous $\mathrm{hFcRn}{ }^{29}$. Cells were seeded in a Transwell system and grown to high electrical

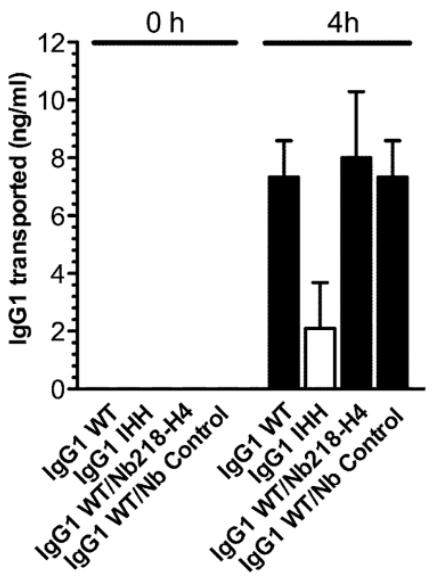

Figure 5 The impact of Nb218-H4 on FcRn-mediated IgG transcellular transport.(D) Polarized human T84 cells were seeded in a Transwell system, and grown to high transcellular resistance $\left(1000-1500 \Omega / \mathrm{cm}^{2}\right)$. Samples of human IgG1-WT or IgG1-IHH were added to the apical reservoir. At time $0 \mathrm{~h}$ and $4 \mathrm{~h}$, medium samples were collected from the basolateral reservoirs. The amounts of IgG transported across the cellular layer were quantified by ELISA. The same assay was performed with WT IgG1 in the presence of a $>15$-fold excess amount of $\mathrm{Nb} 218-\mathrm{H} 4$ or a control $\mathrm{Nb}$. The numbers given represent the mean of triplicates. $n=3$-. All data are presented as mean \pm s.d. 
resistance before recombinantly produced human IgG1 antibodies with specificity for the hapten NIP, a WT variant and a triple mutant (I253A/H310A/H435A; IHH) with reduced FcRn binding, were added to the apical reservoirs. Samples were collected from the basolateral side at time 0 and 4 hours post addition to the apical side. The amounts of IgG1 variants transported across the cellular layers were quantified by ELISA, which demonstrated that IgG1-WT was transported more efficiently than the $\mathrm{IHH}$ triple mutant (Figure 5). When the same experiment was performed in the presence of a 15-fold excess amount of $\mathrm{Nb} 218-\mathrm{H} 4$, no difference in transcytosis of WT IgG1 was detected (Figure 5). Thus, binding of $\mathrm{Nb} 218-\mathrm{H} 4$ to hFcRn does not interfere with transcellular transport of IgG.

\section{Discussion}

FcRn fulfills important functions in IgG-mediated immune surveillance and transport of albumin throughout the body. The increasing appreciation of the impact of FcRn in both homeostatic regulation of the two ligands and pathological conditions related to IgG-mediated autoimmunity has resulted in an intense interest from academia as well as biotech companies. Especially, engineering of FcRn binding and transport of IgG has resulted in development of therapeutic IgGs and IgG-derived Fc fusions with improved serum half-life $\mathrm{e}^{25,26,30-32}$. Furthermore, FcRn blockers, such as peptides that bind tightly to the IgG binding site, inhibit FcRn binding and rescue of circulating IgG, resulting in reduced blood levels of $\operatorname{IgG}^{33}$.

Beside half-life regulation, a large body of evidence has shown that FcRn is expressed in epithelial cells lining the mucosal surfaces where it mediates transport of IgG and IgG-containing immune complexes, which may result in induction of immunity or development of diseases such as colitis ${ }^{12,13,34}$. Furthermore, recent studies have demonstrated a central role of FcRn in processing of IgG-containing immune complexes in dendritic cells for subsequent presentation of antigenic peptides on both MHC class I and $\mathrm{II}^{17,18}$. How these processes are regulated at molecular and cellular levels has not been studied in detail.

The overall goal of this study was to develop and characterize Nbs with specificity for the human form of FcRn. This was motivated by the need for reliable probes for studying the molecular and cellular biology of hFcRn. To this end, a llama was immunized with recombinant soluble hFcRn, followed by the construction of a VHH library and panning on soluble hFcRn. FcRn binds both its ligands strongly only at the acidic $\mathrm{pH}$ found within endosomal compartments. However, the selected $\mathrm{Nbs}$ bound $\mathrm{hFcRn}$ at acidic as well as at neutral $\mathrm{pH}$, exemplified by $\mathrm{Nb} 214-\mathrm{H} 8$, which only showed a 2-fold difference in affinity between pH 6.0 and 7.4. This is important, as the probe will detect $\mathrm{hFcRn}$ at the relevant cellular conditions. None of the anti-FcRn Nbs selected was shown to interfere with $\mathrm{pH}$ dependent binding of IgG and albumin to the receptor, which is a prerequisite for the use of such $\mathrm{Nbs}$ as $\mathrm{FcRn}$ detection probes to address FcRn-mediated ligand binding and transport.

We explored the applications of the Nbs using in vitro binding assays such as ELISA and SPR and found that they could easily be used to detect hFcRn alone or bound to ligands. Also, conjugation of HRP to the minimal Nb214-H8 demonstrated retained ability to bind strongly to $\mathrm{hFcRn}$. Thus, it can be used as a capture reagent or as a detection probe. This was also the case for a dimeric version of $\mathrm{Nb} 214-\mathrm{H} 8$. Furthermore, we conjugated (Nb214-H8) 2 to fluorochromes, and showed that it could be used as cellular tracker to examine FcRn expression and distribution without affecting ligand binding and transport. A notable feature of Nb214-H8 is that it only binds to fully folded hFcRn and as such it only stains hFcRn that has left the endoplasmic reticulum. Besides hFcRn, we have found that Nb218-H4 binds strongly to FcRn from rhesus monkey, weakly to FcRn from pig, and not at all to FcRn from mouse and rat (Supplemental Fig. S3).
In conclusion, we have developed Nbs with specificity for hFcRn. The identified minimal Nbs bind independently of IgG and albumin to hFcRn, and thus are ideally suited for detection and tracking of hFcRn.

\section{Methods}

Production of recombinant hFcRn. Vectors encoding truncated versions of the three ectodomains $(\alpha 1-\alpha 3)$ of mouse, rat and human FcRn HC cDNAs genetically fused to a cDNA encoding the Schistosoma japonicum glutathione $S$-transferase (GST) have been described ${ }^{24,35,36}$. The vectors also contain a cDNA encoding human $\beta_{2}$-microglobulin and the Epstein-Barr virus origin of replication (oriP). Similar, truncated forms of FcRn HC cDNAs from monkey and pig were synthesized by Genscript and subcloned into the vector system using the restriction sites XhoI and HindIII. All FcRn variants were produced in adherent human embryonic kidney $293 \mathrm{E}$ cells, and secreted receptors were purified using a GSTrap column as described ${ }^{37}$. An E. coli produced form of refolded hFcRn was produced and purified as previously described ${ }^{36}$.

Immunizations of llamas. Animal experiments were conducted with the approval of the Ethical committee of the Faculty of Veterinary Medicine (University of Ghent, Belgium, EC number is 2006/076, LA number is 1400088). The animal immunization protocol is based upon the guidelines available for Guanaco and Vicuña (llama species) as described in the Ministerial Decree of 05.03.1999 (for zoo animals) and the guidelines for farm animals used as laboratory animals described in Appendix A of the European convention for the protection of vertebrate animals used for experimental and other scientific purposes, from the European Treaty Series (ETS) 123. In addition, the animal facility was complied with the basic principles of animal care as specified by the Royal Decree 14/08/1986, Law on the Protection and Welfare of Animals (last amended 19/05/2010). This Decree aims to ensure that all species of animal are protected from harm and neglect and are provided with conditions that meet their welfare needs. One llama was immunized with 3 doses of 50-100 $\mu \mathrm{g}$ E. coli produced $\mathrm{hFcRn}{ }^{38}$. Blood was collected 22 days after the last boost. Approximately $1 \mathrm{~g}$ of lymph nodes was collected 4 days after boost 6 .

Phage library construction. PBLs were purified by a Ficoll-Paque density gradient centrifugation (Amersham Biosciences, Uppsala, Sweden) according to the manufacturer's instructions. Next, total RNA was extracted and transcribed into cDNA (Invitrogen superscript III kit). The generated cDNA was used for nested RTPCR to amplify the Nbs encoding gene fragments as previously described ${ }^{39}$, and cloned into the phagemid pAX50. The size of the library after transformation of $E$. coli TG1 was determined as $7-8 \times 10^{8}$ CFUs. $96-100 \%$ of the clones contained a correct size insert as evaluated by colony PCR on 24 randomly picked colonies using M13 reverse and a geneIII primers. Phages were prepared according to standard methods and stored at $4^{\circ} \mathrm{C}$ and/or in $20 \%$ glycerol at $-20^{\circ} \mathrm{C}$.

Phage display selections. Refolded hFcRn was used in selections performed at $\mathrm{pH}$ 5.1. FcRn $(0-10 \mu \mathrm{g} / \mathrm{ml})$ was immobilized on Maxisorp 96 well microtiter plates (Nunc) in PBS at pH 7.4 overnight at $4^{\circ} \mathrm{C}$. After 2 hours blocking with $4 \%$ skimmed milk-PBS, the phages were added and incubated at $37^{\circ} \mathrm{C}$ in $2 \%$ skimmed milk-pH 5.1 CPA buffer (10 mM sodium citrate, $10 \mathrm{mM}$ sodium phosphate, $10 \mathrm{mM}$ sodium acetate, $115 \mathrm{mM} \mathrm{NaCl}, \mathrm{pH}$ 5.1) or in $2 \%$ skimmed milk-PBS in the presence of human $\beta 2$-microglobulin $(12-100 \mu \mathrm{g} / \mathrm{ml}$ ) (Sigma-Aldrich). After 2 hours, the plates were extensively washed with pH 5.1 CPA buffer $-0.05 \%$ Tween 20 or with PBS- $0.05 \%$ Tween 20 and bound phages were eluted using trypsin $(1 \mathrm{mg} / \mathrm{ml})$ for 15 minutes at $37^{\circ} \mathrm{C}$. The eluted phages were amplified in E. coli TG1 and subjected to a second, similar selection. Eluted phages were again rescued in TG1. Individual colonies were picked and grown in 96 well plates. Monoclonal phages were produced by addition of helper phages.

Phage binding ELISA. hFcRn or human $\beta 2$-microglobulin $(0.5 \mu \mathrm{g} / \mathrm{ml})$ were immobilized on Maxisorp ELISA plates (Nunc) at $4^{\circ} \mathrm{C}$ over night, followed by 2 hours blocking using $4 \%$ skimmed milk-PBS. After washing with pH 5.1 or $\mathrm{pH} 7.4$ CPA buffer, $10 \mu \mathrm{l}$ of the different phages diluted in $100 \mu \mathrm{l}$ of $2 \%$ skimmed milk/pH 5.1 or $\mathrm{pH}$ 7.4 CPA buffer and allowed to bind to the immobilized antigen. After 2 hours incubation and washing with $\mathrm{pH} 5.1$ or $\mathrm{pH} 7.4 \mathrm{CPA}$ buffer, $100 \mu \mathrm{l}$ of a horse reddish peroxidase (HRP)-conjugated monoclonal-anti-M13 antibody (GE Healthcare, $1: 10000$ dilutions) in $1 \%$ skimmed milk/pH 5.1 or $\mathrm{pH} 7.4$ CPA buffer was added to the wells for 1 hour. Phage binding was detected by adding $100 \mu$ of $3,3^{\prime}, 5,5^{\prime}$-Tetramethylbenzidine (TMB) substrate (Pierce). The reaction was stopped after 15 minutes and absorbance was measured at $450 \mathrm{~nm}$ using a TECAN Sunrise spectrophotometer (TECAN, Maennedorf, Switzerland). Binding specificity was determined based on OD values compared to controls wells having received an irrelevant phage clone or no phage.

Generation of bivalent Nanobodies. Bivalent $\mathrm{Nb}$ fusions consisting of two copies of the Nb218-H4 or a Nb with irrelevant specificity (Nb203-G8) linked via a 20 amino acid linker (repetitive (GGGGS) ${ }_{4}$ sequence) were constructed by separate PCR reactions (one for the $\mathrm{N}$-terminal and one for the $\mathrm{C}$-terminal), using different sets of primers encompassing parts of the linker and restriction sites MfeI in the N-terminal, or BstEII in the C-terminal. The resulting PCR products were digested with the 
correspondent enzymes and sub-cloned into an expression vector containing C-terminal c-Myc and $\mathrm{His}_{6}$ tags.

Nanobody production and purification. Exponentially growing E. coli cultures (50$1000 \mathrm{ml}$ ) were induced with $1 \mathrm{mM}$ isopropyl-D-thiogalactopyranoside for 4 hours at $37^{\circ} \mathrm{C}$. Bacterial periplasmic space was extracted by centrifugation, freeze/thaw of the pellet and re-suspension in PBS followed by final centrifugation. Nbs were purified from the supernatants i) using TALON beads (Clontech Laboratories, Mountain View, CA) according to the manufacturer recommendation, and dialyzing against PBS, or ii) by using His Trap ${ }^{\mathrm{TM}} \mathrm{FF}$ chromatography columns (GE Healthcare), followed by gel filtration using a HiLoad 16/10 Superdex 75 prep grade column (GE Healthcare). Purity and concentration of the purified Nbs were determined by SDSPAGE analysis.

Nanobody HRP conjugation. Purified Nbs were conjugated to HRP using the EZLink Plus Activated Peroxidase kit (Pierce). Conjugates were purified using a Sephadex-G75 column. $0.5 \mathrm{ml}$ fractions were collected and size-separated by SDSPAGE followed by Coomassie staining. The HRP activity of the fractions was determined by ELISA where $\mathrm{Nb}$ was captured by an immobilized anti c-Myc tag antibody (monoclonal, mIgG1; Serotec) and detection was carried out using TMB substrate.

Nanobody Alexa labeling. Purified Nbs were adjusted to $\mathrm{pH} 8.3$ with $1 / 10$ volume $1 \mathrm{M} \mathrm{NaHCO}_{3}$ and directly labeled with amine-reactive AlexaFluor647 or with AlexaFluor488 (Invitrogen). A 10-fold M excess of dye over protein was used. Labeling reaction was quenched using $1 / 10$ volume $1.5 \mathrm{M}$ Tris- $\mathrm{HCl}$. Desalting was performed on PD MiniTRap G25 spin columns (GE Healthcare). Human albumin (Sigma-Aldrich) was conjugated to Alexa647 using Alexa Fluor 647 Protein Labeling Kit (Invitrogen). The concentration of the conjugated proteins was estimated both spectrophotometrically (Nanodrop, Thermo Scientific) and by densitometry analyses of scanned Coomassie-stained SDS-PAGE.

FcRn binding ELISA. Wells were coated with $100 \mu \mathrm{l}$ of an anti-Myc antibody (Serotec) at $1.0 \mu \mathrm{g} / \mathrm{ml}$, and incubated overnight at $4{ }^{\circ} \mathrm{C}$. They were then blocked for 1 hour with $4 \%$ skimmed milk (Acumedia) and washed four times with PBS/0.005\% Tween 20 (PBS/T) pH 7.4. Nb candidates $(2 \mu \mathrm{g} / \mathrm{ml})$ in $4 \%$ skimmed milk/PBS/T were added to the wells. After incubation for 1 hour, the wells were washed four times with $\mathrm{PBS} / \mathrm{T} \mathrm{pH}$ 7.4. GST-tagged $\mathrm{hFcRn}(0.5 \mu \mathrm{g} / \mathrm{ml})$ pre-incubated with an HRP-

conjugated polyclonal anti-GST antibody from goat (1:5000; GE Healthcare) in $4 \%$ skimmed milk PBS/T pH 7.4 was added to each well and incubated for 1 hour. Following four washes with PBS/T pH 7.4, $100 \mu \mathrm{l}$ of ABTS $/ \mathrm{H}_{2} \mathrm{O}_{2}$ (Sigma-Aldrich) was added and absorbance at $405 \mathrm{~nm}$ was measured using a TECAN Sunrise spectrophotometer (TECAN). The assay was repeated with $\mathrm{PBS} / \mathrm{T} \mathrm{pH} 6.0$, and with serial dilutions of Nbs $(0.016-4.0 \mu \mathrm{g} / \mathrm{ml})$. To determine species cross-reactivity, the ELISA was performed using soluble GST-tagged forms of mouse, rat, pig, monkey FcRn. Alternatively, titrated amounts of IgG or human albumin were coated in wells followed by GST-tagged hFcRn and detection using HRP-conjugated $\mathrm{Nb} 218-\mathrm{H} 4$.

Human $\beta 2$-microglobulin (Abcam) was coated $(5 \mu \mathrm{g} / \mathrm{ml})$ and incubated overnight at $4{ }^{\circ} \mathrm{C}$. Wells were then blocked in with $4 \%$ skimmed milk for 1 hour and washed four times with PBS/T pH 6.0. Nb candidates $(2 \mu \mathrm{g} / \mathrm{ml})$ were pre-incubated with an HRPconjugated anti c-Myc antibody (mIgG1, Serotec) in 4\% skimmed milk/PBS/T and added to the wells. After incubation for 1 hour, the wells were washed four times with $\mathrm{PBS} / \mathrm{T}$ pH 6.0. $100 \mu \mathrm{l}$ of the substrate TMB (Calbiochem) was added to each well and the absorbance was measured at $620 \mathrm{~nm}$ using a TECAN Sunrise spectrophotometer (TECAN). An anti-human $\beta 2$-microglobulin antibody ( $1: 2000$; Sigma-Aldrich) was included as a positive control and binding visualized using HRP-conjugated protein G (Calbiochem)

Detection of llama IgG antibodies directed towards hFcRn in pre- and postimmunization sera was done similarly, using mouse-anti-llama antibodies ${ }^{40}$ followed by an HRP-conjugated rabbit anti-mouse antibody (Dako, DK).

SPR. SPR experiments were carried out using a Biacore 3000 instrument (GE Healthcare). Flow cells of CM5 sensor chips were coupled with hFcRn-GST ( $~ 800 \mathrm{RU})$, human IgG or human albumin $(\sim 1000 \mathrm{RU})$ using amine coupling chemistry as described by the manufacturer. The coupling was performed by injecting $10 \mu \mathrm{g} / \mathrm{ml}$ of the receptor in $10 \mathrm{mM}$ sodium acetate $\mathrm{pH} 5.0$ (GE healthcare). For all experiments, phosphate buffer $(67 \mathrm{mM}$ phosphate buffer, $0.15 \mathrm{M} \mathrm{NaCl}, 0.005 \%$ Tween 20) at pH 6.0 or pH 7.4, or HBS-EP buffer (0.01 M HEPES, $0.15 \mathrm{M} \mathrm{NaCl}$, 3 mM EDTA, 0.005\% surfactant P20) at pH 7.4 (GE Healthcare) were used as running buffer and dilution buffer.

For competitive binding analyses, $\mathrm{hFcRn}$ was pre-incubated with the $\mathrm{Nb}$ candidates and injected over immobilized human IgG1 or albumin at pH 6.0. All injections were done using a constant flow rate of $10 \mu \mathrm{l} / \mathrm{min}$ at $25^{\circ} \mathrm{C}$. For kinetic analysis, serial dilutions (500-15 nM) of monomeric Nb218-H4 were injected at a flow rate of $50 \mu \mathrm{l} /$ min at $25^{\circ} \mathrm{C}$ over immobilized $\mathrm{hFcRn}$ at $\mathrm{pH} 6.0$ or $\mathrm{pH}$ 7.4. The resulting sensorgrams were analyzed using the BIAevaluation 4.1 software using Langmuir 1: 1 binding model and the steady state affinity binding models to derive binding kinetics. In all experiments, data were zero adjusted and the reference cell subtracted.

Flow cytometry. The human monocytic cell line THP-1 (ATCC) was cultured in RPMI 1640 (BioWhittaker, Belgium) supplemented with $4 \mathrm{mM}$ L-glutamine, $100 \mathrm{U} / \mathrm{ml}$ penicillin (Integro, The Netherlands), $0.1 \mathrm{mM}$ nonessential amino acids and $10 \%$ foetal calf serum (FCS) (Integro). $1 \times 10^{6}$ cells were washed three times with PBS followed by centrifugation at $800 \mathrm{rpm}$, re-suspended and added to 96-well plates (Nunc). Cells were fixed with 3\% paraformaldehyde (PFA) (Sigma-Aldrich) for $30 \mathrm{~min}$, permeabilized with $0.1 \%$ saponin and then blocked with $2 \%$ bovine serum albumin (BSA)/PBS (Sigma-Aldrich) for 1 hour. Following three washes with PBS/ BSA, Alexa488 or Alexa647 conjugated (Nb218-H4) $)_{2}$ or a bivalent control $\mathrm{Nb}(1 \mu \mathrm{g} /$ $\mathrm{ml})$ diluted in PBS/BSA were added. Following 1 hour staining at $4^{\circ} \mathrm{C}$, cells were again washed as described above and analyzed by flow cytometry.

Immunofluorescence and confocal laser scanning microscopy. HeLa cells were grown on coverslips to $70 \%$ confluence in complete DMEM and transfected with vectors encoding $\mathrm{hFcRn}$-RFP and human $\beta 2$-microglobulin or hFcRn-RFP alone, using FuGENE 6.0 transfection reagent according to the recommendations from the manufacturer (Roche). One day post transfection, cells were incubated with $2 \mu \mathrm{g} / \mathrm{ml}$ fluorescently conjugated (Nb218-H4) 2 (Alexa 488), an Nb control (Alexa 488), or human albumin (Alexa 647, all fluorochromes from Invitrogen) for 1 hour. Cells were then washed in PBS, fixed in 3\% PFA and mounted onto object-glasses with Mowiol (Sigma-Aldrich). Confocal images were acquired on an Olympus FluoView 1000 microscope equipped with a PlanApo 0/1.10 oil objective (Olympus, Hamburg, Germany). Fluorochromes were exited with $488 \mathrm{~nm}$ Argon, $543 \mathrm{~nm}$ and $647 \mathrm{~nm}$ $\mathrm{HeNe}$ lasers. All image acquisition was done by sequential line scanning to eliminate bleed-through. Images were processed with Image (NIH, USA) and assembled in Adobe Illustrator (Adobe systems Inc., CA, USA)

Transepithelial IgG transport. The human T84 cell line (ATCC) was maintained in DMEM and HAMs F-12 medium (1:1) supplied with 10\% FCS (Intebro b.v.), $2.5 \mathrm{mM} \mathrm{L}$-glutamine, $25 \mu \mathrm{g} / \mathrm{ml}$ streptomycin and $25 \mathrm{U} / \mathrm{ml}$ penicillin (all from BioWhittaker). $3 \times 10^{6}$ cells were seeded on Transwell filters with $0.4 \mu \mathrm{m}$ pore size (Corning Costar, MA, USA) and transepithelial resistance was measured daily using MILLICELL-ERS volt-ohm meter (MILLIPORE). The cells were grown as monolayers for 4-6 days until the resistance reached $1000-1500 \Omega / \mathrm{cm}^{2}$. Growth medium were exchanged daily. Cells were then cultured in HBSS + buffer for 1 hour before equal amounts ( $66 \mathrm{nM}$ ) of recombinant human IgG1-WT and IgG1 IHH (I253A/H310A/H435A) with specificity for 5-iodo-4-hydroxy-3-nitro-phenylacetyl (NIP) was added to individual apical reservoirs. At time 0 and $4 \mathrm{~h}$ post addition, samples were collected from the basolateral reservoirs, and the amounts of IgG present quantified by ELISA. The same assay was performed in the presence of $1 \mu \mathrm{M}$ of the Nb218-H4 or an $\mathrm{Nb}$ with irrelevant specificity, both added to the apical side 20 minutes before the IgG antibodies were provided.

1. Rodewald, R. \& Kraehenbuhl, J. P. Receptor-mediated transport of IgG. J Cell Biol 99, 159s-164s (1984).

2. Simister, N. E. \& Mostov, K. E. An Fc receptor structurally related to MHC class I antigens. Nature 337, 184-187 (1989).

3. Roopenian, D. C. \& Akilesh, S. FcRn: the neonatal Fc receptor comes of age. Nature reviews. Immunology 7, 715-725 (2007)

4. Ward, E. S. \& Ober, R. J. Chapter 4: Multitasking by exploitation of intracellular transport functions the many faces of FcRn. Advances in immunology 103, 77-115 (2009).

5. Stapleton, N. M. et al. Competition for FcRn-mediated transport gives rise to short half-life of human IgG3 and offers therapeutic potential. Nature communications 2, 599 (2011)

6. Kim, J. K. et al. Mapping the site on human IgG for binding of the MHC class Irelated receptor, FcRn. European journal of immunology 29, 2819-2825 (1999).

7. Chaudhury, C. et al. The major histocompatibility complex-related Fc receptor for IgG $(\mathrm{FcRn})$ binds albumin and prolongs its lifespan. The Journal of experimental medicine 197, 315-322 (2003).

8. Morell, A., Terry, W. D. \& Waldmann, T. A. Metabolic properties of IgG subclasses in man. J Clin Invest 49, 673-680 (1970).

9. Peters, T., Jr. Serum albumin. Advances in protein chemistry 37, 161-245 (1985)

10. Montoyo, H. P. et al. Conditional deletion of the MHC class I-related receptor FcRn reveals the sites of IgG homeostasis in mice. Proceedings of the National Academy of Sciences of the United States of America 106, 2788-2793 (2009).

11. Akilesh, S., Christianson, G. J., Roopenian, D. C. \& Shaw, A. S. Neonatal FcR expression in bone marrow-derived cells functions to protect serum IgG from catabolism. J Immunol 179, 4580-4588 (2007).

12. Kobayashi, K. et al. An FcRn-dependent role for anti-flagellin immunoglobulin G in pathogenesis of colitis in mice. Gastroenterology 137, 1746-1756 e1741 (2009).

13. Spiekermann, G. M. et al. Receptor-mediated immunoglobulin G transport across mucosal barriers in adult life: functional expression of $\mathrm{FcRn}$ in the mammalian lung. The Journal of experimental medicine 196, 303-310 (2002).

14. Bitonti, A. J. et al. Pulmonary delivery of an erythropoietin Fc fusion protein in non-human primates through an immunoglobulin transport pathway. Proc Natl Acad Sci U S A 101, 9763-9768 (2004).

15. Story, C. M., Mikulska, J. E. \& Simister, N. E. A major histocompatibility complex class I-like Fc receptor cloned from human placenta: possible role in transfer of immunoglobulin G from mother to fetus. J Exp Med 180, 2377-2381 (1994).

16. Firan, M. et al. The MHC class I-related receptor, FcRn, plays an essential role in the maternofetal transfer of gamma-globulin in humans. International immunology 13, 993-1002 (2001). 
17. Qiao, S. W. et al. Dependence of antibody-mediated presentation of antigen on FcRn. Proceedings of the National Academy of Sciences of the United States of America 105, 9337-9342 (2008).

18. Baker, K. et al. Neonatal Fc receptor for $\operatorname{IgG}(\mathrm{FcRn})$ regulates cross-presentation of IgG immune complexes by CD8-CD11b + dendritic cells. Proceedings of the National Academy of Sciences of the United States of America 108, 9927-9932 (2011).

19. Burmeister, W. P., Huber, A. H. \& Bjorkman, P. J. Crystal structure of the complex of rat neonatal Fc receptor with Fc. Nature 372, 379-383 (1994).

20. West, A. P., Jr. \& Bjorkman, P. J. Crystal structure and immunoglobulin G binding properties of the human major histocompatibility complex-related Fc receptor(,). Biochemistry 39, 9698-9708 (2000).

21. Andersen, J. T. et al. Structure-based mutagenesis reveals the albumin-binding site of the neonatal Fc receptor. Nature communications 3, 610 (2012).

22. Andersen, J. T., Dee Qian, J. \& Sandlie, I. The conserved histidine 166 residue of the human neonatal $\mathrm{Fc}$ receptor heavy chain is critical for the $\mathrm{pH}$-dependent binding to albumin. European journal of immunology 36, 3044-3051 (2006).

23. Chaudhury, C., Brooks, C. L., Carter, D. C., Robinson, J. M. \& Anderson, C. L. Albumin binding to FcRn: distinct from the FcRn-IgG interaction. Biochemistry 45, 4983-4990 (2006).

24. Andersen, J. T., Daba, M. B., Berntzen, G., Michaelsen, T. E. \& Sandlie, I. Crossspecies binding analyses of mouse and human neonatal $\mathrm{Fc}$ receptor show dramatic differences in immunoglobulin $\mathrm{G}$ and albumin binding. The Journal of biological chemistry 285, 4826-4836 (2010).

25. Andersen, J. T. \& Sandlie, I. The versatile MHC class I-related FcRn protects IgG and albumin from degradation: implications for development of new diagnostics and therapeutics. Drug metabolism and pharmacokinetics 24, 318-332 (2009).

26. Kuo, T. T. et al. Neonatal Fc receptor: from immunity to therapeutics. Journal of clinical immunology 30, 777-789 (2010).

27. Van Bockstaele, F., Holz, J. B. \& Revets, H. The development of nanobodies for therapeutic applications. Curr Opin Investig Drugs 10, 1212-1224 (2009).

28. Claypool, S. M., Dickinson, B. L., Yoshida, M., Lencer, W. I. \& Blumberg, R. S. Functional reconstitution of human FcRn in Madin-Darby canine kidney cells requires co-expressed human beta 2-microglobulin. The Journal of biological chemistry 277, 28038-28050 (2002).

29. Dickinson, B. L. et al. Bidirectional FcRn-dependent IgG transport in a polarized human intestinal epithelial cell line. The Journal of clinical investigation 104, 903-911 (1999).

30. Zalevsky, J. et al. Enhanced antibody half-life improves in vivo activity. Nature biotechnology 28, 157-159 (2010).

31. Andersen, J. T. et al. Anti-carcinoembryonic antigen single-chain variable fragment antibody variants bind mouse and human neonatal Fc receptor with different affinities that reveal distinct cross-species differences in serum half-life. The Journal of biological chemistry 287, 22927-22937 (2012).

32. Petkova, S. B. et al. Enhanced half-life of genetically engineered human IgG1 antibodies in a humanized FcRn mouse model: potential application in humorally mediated autoimmune disease. International immunology 18, 1759-1769 (2006).

33. Mezo, A. R. et al. Reduction of IgG in nonhuman primates by a peptide antagonist of the neonatal Fc receptor FcRn. Proceedings of the National Academy of Sciences of the United States of America 105, 2337-2342 (2008).
34. Yoshida, M. et al. Human neonatal Fc receptor mediates transport of IgG into luminal secretions for delivery of antigens to mucosal dendritic cells. Immunity 20, 769-783 (2004)

35. Andersen, J. T. et al. Extending half-life by indirect targeting of the neonatal $\mathrm{FC}$ receptor $(\mathrm{FcRn})$ using a minimal albumin binding domain. The Journal of biological chemistry 286, 5234-5241 (2011).

36. Andersen, J. T. et al. Ligand binding and antigenic properties of a human neonatal Fc receptor with mutation of two unpaired cysteine residues. The FEBS journal 275, 4097-4110 (2008)

37. Berntzen, G. et al. Prolonged and increased expression of soluble Fc receptors, IgG and a TCR-Ig fusion protein by transiently transfected adherent $293 \mathrm{E}$ cells. Journal of immunological methods 298, 93-104 (2005).

38. Andersen, J. T. et al. A strategy for bacterial production of a soluble functional human neonatal Fc receptor. J Immunol Methods 331, 39-49 (2008).

39. Roovers, R. C. et al. Efficient inhibition of EGFR signaling and of tumour growth by antagonistic anti-EFGR Nanobodies. Cancer Immunol Immunother 56, 303-317 (2007)

40. Daley, L. P., Gagliardo, L. F., Duffy, M. S., Smith, M. C. \& Appleton, J. A. Application of monoclonal antibodies in functional and comparative investigations of heavy-chain immunoglobulins in new world camelids. Clin Diagn Lab Immunol 12, 380-386 (2005).

\section{Acknowledgments}

We are grateful to Sathiaruby Sivaganesh and Peggy Willemarck for excellent technica assistance. This work was supported by the Norwegian Research Council (Grant no. 179573/V40; J.T.A.), South-Eastern Norway Regional Health Authority (Grant no. 39375; J.T.A.) and Ablynx NV was supported by Institute for the Promotion of Innovation by Science and Technology in Flanders from the Flemish government (Grant no. IWT70050).

\section{Author contributions}

J.T.A., S.F., M.G., O.J.B.L., P.V. H.J.H., M.S. and I.S. designed research; J.T.A., S.F., M.G., D.P., A.S. and O.J.B.L. performed research; J.T.A., S.F., M.G., O.J.B.L. and I.S. analyzed data; J.T.A. and I.S wrote the paper.

\section{Additional information}

Supplementary information accompanies this paper at http://www.nature.com/ scientificreports

Competing financial interests: The authors declare no competing financial interests. License: This work is licensed under a Creative Commons Attribution-NonCommercial-NoDerivs 3.0 Unported License. To view a copy of this license, visit http://creativecommons.org/licenses/by-nc-nd/3.0/

How to cite this article: Andersen, J.T. et al. Selection of Nanobodies that Target Human Neonatal Fc Receptor. Sci. Rep. 3, 1118; DOI:10.1038/srep01118 (2013) 